\title{
COMPRENDRE LES COMPORTEMENTS DES POPULATIONS EN SITUATION DE RISQUE NATUREL DANS LES PAYS EN DÉVELOPPEMENT. L' EXEMPLE DE DOUALA (CAMEROUN)
}

\author{
Gille Baustert TCHOUNGA
}

\begin{abstract}
Résumé
Dans les pays en développement, la gestion des risques naturels par les ménages met en exergue des réactions sociales dont la compréhension reste complexe. Comment le risque est-il pensé dans un contexte de précarité ? Qu'est ce qui détermine les actions des ménages en situation de risque naturel ? À partir des enquêtes de terrain et des modèles théoriques en sciences sociales (capabilités et motivation à la protection), cet article relève les déterminants de l'adoption des comportements préventifs en situation de catastrophe naturelle dans la ville de Douala. Plusieurs éléments contextuels motivent les actions des ménages dans les zones à risque, l'analyse conduit à deux attitudes adoptées à la suite du processus perception-représentation-comportement : les initiatives protectrices liés à la minimisation du risque et celles non protectrices liées au déni et au sentiment de sécurité. Le renforcement de la communication sur les risques naturels constitue une dimension indispensable à considérer dans la gestion durable des territoires exposés aux risques naturels.
\end{abstract}

Mots-clés

comportements, perceptions, pays en développement, représentations, risques naturels

\begin{abstract}
In developing countries, the management of natural risks by households highlights social reactions whose understanding remains complex. How is risk thought in a context of precariousness? What determines the actions of households in situations of natural risk? Based on field surveys and theoretical models in the social sciences (capabilities and motivation for protection), this article highlights the determinants of the adoption of preventive behavior in a natural disaster situation in the city of Douala. Several contextual elements motivate the actions of households in risk areas, the analysis leads to two attitudes adopted following the perception-representation-behavior process: protective initiatives related to risk minimization and non-protective initiatives related to denial and to the feeling of security. Strengthening communication on natural risks is an essential dimension in the sustainable management of areas exposed to natural risks.
\end{abstract}

Keywords

behaviors, perception, developing countries, representation, natural risk

\section{INTRODUCTION}

Les pays en développement font face à de nombreuses catastrophes naturelles dont l'ampleur des dégâts est liée à la concentration des populations dans les sites dangereux. Ces pays comme ceux développés, sont exposés à des menaces naturelles comparables en intensité, en nombre et aussi en formes (Bétard et Fort, 2014). Cependant, les conséquences des évènements dommageables sont plus alarmantes dans les villes des pays en développement, cette disparité trouve une justification dans les politiques de gestion des risques naturels. $\mathrm{La}$ gestion des évènements naturels dommageables au Sud reste globalement penchée sur une vision techniciste, elle porte sur des aménagements urbains visant la maitrise de l'aléa. Avec l'urbanisation galopante dans les zones à risque et l'augmentation des vulnérabilités urbaines, ce mode de gestion des risques a montré ses limites, les études se penchent dorénavant sur le renforcement de la culture du risque pour réduire la vulnérabilité des enjeux exposés, contribuant de ce fait à la durabilité du territoire (Hallegate et al., 2017 ; Chouari, 2019).

Les risques naturels restent mal évalués dans les pays en développement, les aspects touchant les enjeux vulnérables sont ignorés, ces informations sont parfois indisponibles dans certains pays (Gondard-Delcroix et Rousseau, 2004 ; Bétard et Fort, 2014). Il est fréquent que des populations vulnérables se réinstallent dans les sites affectés au lendemain d'une ca- 
tastrophe naturelle, le niveau d'acceptabilité sociale des risques naturels y reste élevé, malgré les pertes humaines enregistrées (Thouret, 2003 ; Betto, 2018). Comment expliquer les différences de comportement pour les populations exposées à des aléas naturels de même intensité ? Qu'est ce qui peut encourager un individu à s'installer dans une zone dangereuse tout en ayant conscience des pertes potentielles en situation de catastrophe naturelle ? Qu'est ce qui détermine l'adoption des comportements préventifs avant, pendant ou après une crise ?

La ville de Douala au Cameroun (Afrique Centrale) est menacée par les inondations et les mouvements de terrain (Olinga, 2012 ; Amanejieu, 2018), leur fréquence va croissante depuis les années 1980, alors qu'on enregistre une baisse des précipitations depuis 1971 à l'ordre de $723,8 \mathrm{~mm}$, ce qui implique une baisse du nombre de jours de pluies (Tchiadeu et Olinga ; 2012). Cette forte occurrence des risques naturels est liée à une urbanisation incontrôlée qui a poussé à la construction des territoires inhérents aux aléas naturels (Mbaha et al., 2013 ; Saha et al, 2018). L'ampleur des dégâts observés dans ces territoires pousse à s'interroger sur les comportements des ménages exposés et surtout leur prise en compte dans les politiques urbaines. À partir d'un cadre théorique (modèle des capabilités de Sen, 1993 et modèle de la motivation à la protection de Rogers, 1983) et d'une approche socio-économique dans la ville de Douala, les résultats développés dans cette étude répondent aux questions évoquées ci-dessus.

L'enjeu de cette recherche réside dans l'amélioration de la résilience sociale dans les territoires à risque et interroge le rapport au risque des ménages à Douala. La mise en perspective des comportements sociaux à travers les processus perceptifs constitue une piste novatrice dans la prévention des risques naturels. Cette recherche privilégie une approche de gestion des risques naturels par la réduction des vulnérabilités sociales, elle favorise le renforcement de la culture du risque et la mise en place des politiques locales pour une gestion intégrée des risques naturels.

\section{CADRAGE DE L'ÉTUDE}

\section{A. Caractéristiques de la zone d'étude}

Cette recherche est menée dans la ville de Douala (Cameroun), c'est une ville-port, principale porte maritime du Cameroun depuis l'époque coloniale, elle constitue un pôle économique pourvoyeur d'emplois. La prospérité économique depuis l'époque coloniale a conduit à un afflux massif des populations à la recherche de meilleures conditions de vie, la population passe d'un effectif estimé à 15000 en 1884 pour atteindre environ 3000000 aujourd'hui (Tchounga, 2020). L'urbanisation s'y est effectuée par vagues concentriques autour du noyau originel et des villages situés le long des axes routiers, au point de coloniser aujourd'hui des espaces impropres à l'habitat (Olinga, 2012).

La crise socio-économique des années 1990 a causé un véritable déficit en logements résidentiels à Douala, les migrants victimes de cette situation ont progressivement colonisé les zones à risque, ce fut le début du développement de la ville informelle et de la construction des territoires du risque (Mbaha et al., 2013). C'est ainsi que les versants instables, les vallées inondables et même les espaces à mangrove ont connu une urbanisation sans précèdent (Mainet, 2005). Dans ces zones de forte concentration démographique et de précarité ambiante, le risque naturel est en deçà des préoccupations vitales des populations exposées, elles sont plus occupées à améliorer leurs conditions de vie et s'accoutument davantage aux risques naturels qu'elles côtoient au quotidien (Tadonki, 1999).

À Douala, les catastrophes naturelles menacent les hommes et les activités économiques, elles sont en augmentation et restent inféodées à la dynamique urbaine (Olinga, 2012 ; Mounde, 2019). Les inondations et les mouvements de terrain y ont causé des dommages importants depuis les années 1980 : environ 45 morts, 58 habitations détruites et de nombreux dégâts matériels non évalués (Tchounga, 2020). L'ampleur des dégâts est liée à la forte concentration démographique dans les quartiers précaires, principaux secteurs affectés par les risques naturels (Amanejieu, 2018). Parlant de la réalité des risques naturels, la ville de Douala est principalement menacée par les inondations et les mouvements de terrain (Figure 1).

L'inondation est le risque naturel le plus présent à Douala, sa délimitation montre que les quartiers précaires sont plus exposés (Fogwe et Tchotsoua, 2007 ; Saha et al., 2018). Les mouvements de terrain sont localisés sur la rive continentale gauche de la ville, ils sont amplifiés par l'urbanisation non 
dans les pays en développement. L'exemple de Douala (Cameroun)

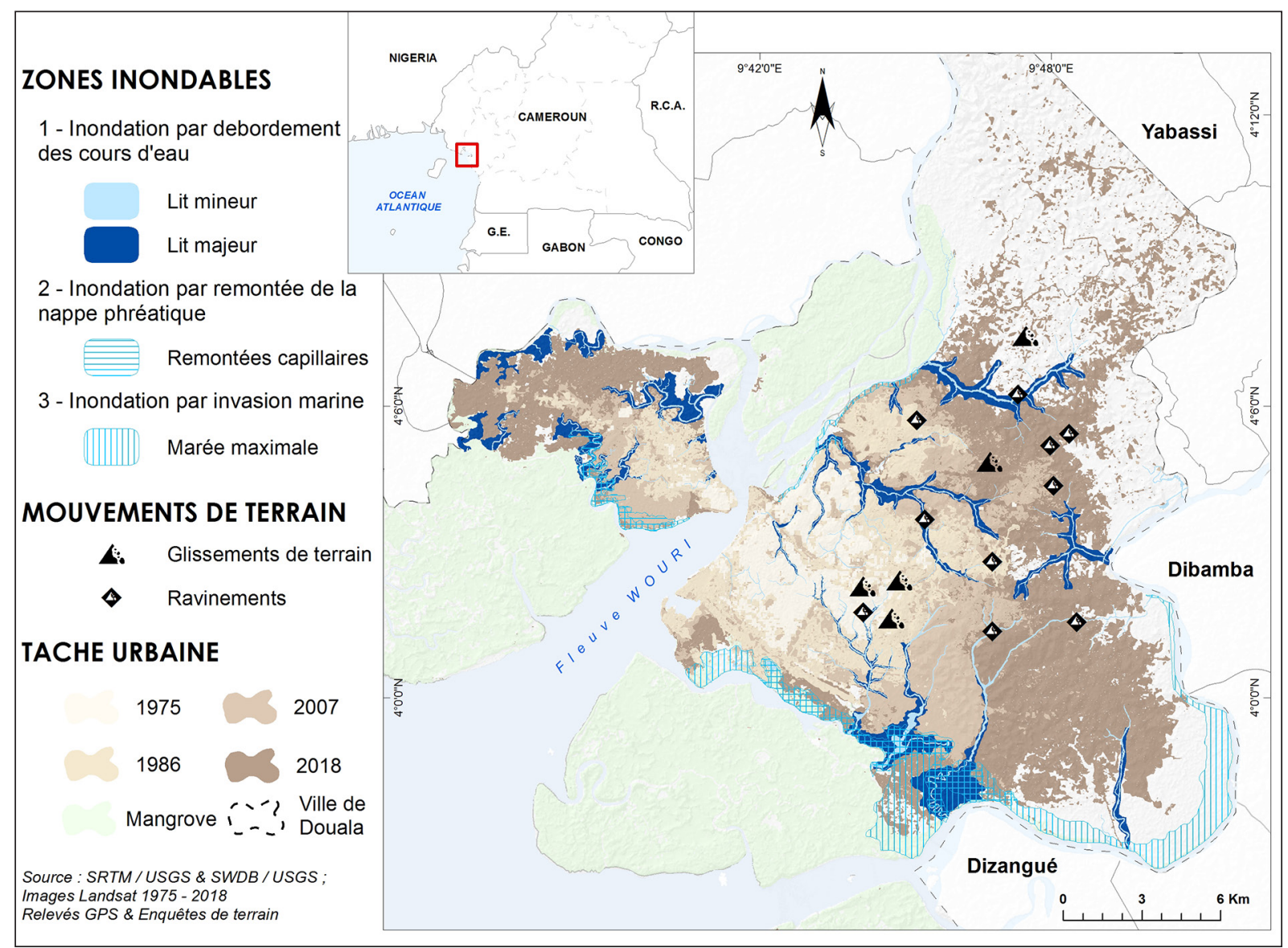

Figure 1. Dynamique urbaine et risques naturels à Douala

contrôlée au point où Douala compte aujourd'hui 11 ravins dont la dynamique génère plusieurs éboulements mettant en danger les enjeux urbains (Meva'a et Fouda, 2018). L'exposition au risque est liée aux territoires défavorisés, ce sont des espaces naturels dont l'urbanisation récente a contribué au dysfonctionnement de l'équilibre environnemental, d'où la récurrence des catastrophes naturelles (Olinga, 2012 ; Bruckmann et al., 2019).

\section{B. Définitions conceptuelles et théoriques}

Le risque naturel est le résultat de la conjonction d'un aléa naturel et d'une société vulnérable (Saha et al., 2018), les conceptions actuelles privilégient une analyse des vulnérabilités sociales dans l'objectif de réduire l'impact des catastrophes naturelles (Quenault, 2015 ; Tchékémian, 2018). L'évaluation de la vulnérabilité fait état de la capacité des enjeux à faire face à la manifestation des aléas naturels, elle permet de définir les niveaux de préparation et de gestion de crise, en d'autres termes les comportements sociaux en situation de risque naturel (Maichanou, 2014). Des analyses plus récentes substituent la vulnérabilité à la résilience (Wisner et al., 2004 ; Barocca et al., 2013 ; Lallau et al., 2018 ; Cantin et Guézo, 2020), particulièrement la vulnérabilité sociale qui fait état de la préparation et de la gestion de crise par une société, d'où son lien direct avec le concept de résilience. La vulnérabilité sociale cesse d'être considérée comme la propension à subir des dommages, elle évalue la capacité de réponse des sociétés en situation de risque (Betard et Fort, 2014 ; Lallau et al., 2018 ).

La résilience d'une société face aux risques naturels s'exprime par sa capacité à faire face, à s'adapter et à trouver un état initial au lendemain de la catastrophe, elle met en exergue les problématiques liées à la gestion du risque (Benitez et Reghezza, 2018). Une telle analyse relève les comportements des populations en situation de risque, de nombreux facteurs cognitifs influencent de ce fait ces comportements sociaux, ces actions sont toutefois liées aux perceptions de l'environnement.

Pour Bonnet (2014), la perception est la «capacité qui permet à un organisme de guider ses actions et de connaitre son environnement sur la base des informations fournies par les sens ». Les percep- 
tions conduisent à la qualification du risque naturel (surestimation ou sous-estimation), et expliquent les constructions mentales qui se forment à leur propos (Bercera et al., 2013). Guidés par la perception, les comportements sociaux conduisent à l'action ou à l'inaction face aux risques naturels en fonction des représentations qui en découlent. La représentation est le résultat d'un processus mental ayant pour élément déclencheur les informations perçues, c'est à partir de ce processus que l'individu construit le réel et lui attribue un sens; elle influence les pratiques sociales en situation de risque (Tchékémian, 2020). Dans ce contexte, deux modèles constituent notre cadre théorique de référence : le modèle de la motivation à la protection (Rogers, 1983) et le modèle des capabilités (Sen, 1993).

Le modèle de la motivation à la protection de Rogers (1983) permet d'expliquer les raisons d'adoption de comportements préventifs dans les zones à risque, il est lié aux sources d'information disponibles ou reconnues et à la perception de la vulnérabilité individuelle, faisant appel aux études comportementales. Ce modèle énonce une approche coût-bénéfice, l'individu en situation de risque évalue la menace et les méthodes de protection, choisit par la suite d'agir ou pas selon les récompenses au bout de chaque action (Bercera et al. 2013 ; Kouabenan, 2007 ; Grothmann et Reusswig, 2006). Elle avance l'idée selon laquelle la motivation à la protection est conditionnée par deux dimensions : L'évaluation de la menace ou perception du risque et l'évaluation de la capacité à composer avec le risque, après cette phase d'évaluation le ménage choisira une action en fonction de ses capacités d'action.

Le modèle des capabilités de Sen (1993) met en exergue la vulnérabilité des populations à partir de l'analyse de leur bien-être. Une analyse qui selon des études va converger vers l'évaluation des capacités d'action des ménages. Gondard-Delcroix et Rousseau (2004) relèvent la capacité des ménages ruraux de Madagascar à faire face aux situations à risque ; Lallau (2008) à travers un cadre conceptuel associant risques, capabilités, vulnérabilité et résilience, inspiré par l'approche des capabilités, étudie les pratiques de gestion des risques accessibles aux agriculteurs pauvres ; Bidou et Droy (2013) évaluent les capabilités à travers l'analyse du lien pauvreté et vulnérabilité sur 204 ménages à Madagascar ; Benitez et Reghezza (2018) ap- pliquent ce modèle théorique aux ménages à Haïti. D'après ce modèle d'analyse économique, les ménages disposent du même espace de fonctionnement (possibilités d'actions), mais réagissent différemment face aux risques à cause de la limite des moyens d'actions disponibles pour activer une action préventive. Les données utilisées dans ce modèle sont basées sur des indicateurs, regroupés dans trois dimensions : le capital physique (biens matériels), le capital social (éducation, santé, taille des ménages, assainissement, accessibilité, genre) et le capital économique (revenu).

Par opposition à l'approche structurelle centrée sur l'aléa naturel, cette étude privilégie l'approche par la vulnérabilité sociale pour la promotion des territoires résilients. La combinaison des modèles d'analyse cognitive et économique réside dans leur fort potentiel à mettre en relief le rapport des ménages au risque et à orienter les décideurs vers la mise en place de nouvelles politiques locales de gestion des risques axées sur la réduction des vulnérabilités sociales.

\section{Présentation de la méthodologie de collecte des données}

Cette étude vise à comprendre les comportements des populations exposées aux inondations et aux mouvements de terrain, tout en analysant le processus allant de la perception de l'information dédiée aux évènements naturels à leur impact sur la réalité urbaine. Il s'agit de relever l'ensemble des éléments influençant la perception des risques naturels par les populations et mesurer leur rôle dans l'adoption des comportements préventifs. La méthode géohistorique a été appliquée pour relever les évènements naturels les plus catastrophiques et les principales zones affectées afin d'y effectuer les enquêtes (Tableau 1). Cette analyse s'est au préalable appuyé sur les travaux de cartographie et d'analyse historique des inondations (Zogning et al., 2013 ; Bruckmann et al., 2019) et des mouvements de terrain dans la ville de Douala (Olinga, 2012).

Des enquêtes de terrain ont été effectuées dans le cadre d'une recherche doctorale (2016 - 2019) sur un échantillon de 765 ménages résidant principalement dans les zones à risque à Douala, le choix des zones d'enquête est opéré en fonction des antécédents catastrophiques. Au total, neuf quartiers ont été sélectionnés pour la collecte des données sur 
dans les pays en développement. L’exemple de Douala (Cameroun)

\begin{tabular}{|l|l|l|l|}
\hline Périodes & Événements & Lieux les plus affectés & Dommages \\
\hline $03 / 08 / 2000$ & Inondations & $\begin{array}{l}\text { Tergal, Nkolmintag, Bois } \\
\text { des Singes, Dibom, Soboum, } \\
\text { Bilongué, Makepe Missoke, } \\
\text { Mambanda, Beedi, Boko }\end{array}$ & $\begin{array}{l}6 \text { morts, } 11 \text { blessés et } \\
\text { plus de } 1200 \text { sans-abris. } \\
23 \text { ponts endommagés }\end{array}$ \\
\hline $29 / 08 / 2005$ & Mouvement de terrain & Madagascar & $\begin{array}{l}3 \text { morts, } 1 \text { habitation } \\
\text { détruite }\end{array}$ \\
\hline $19 / 08 / 2011$ & Mouvement de terrain & $\begin{array}{l}\text { Ndogbong } \\
\text { détruites }\end{array}$ \\
\hline $20 / 06 / 2015$ & Inondations & $\begin{array}{l}\text { Cité Berge, Youpwé, Beedi, } \\
\text { Bois des Singes, Madagas- } \\
\text { car, Makepe Missoke, Boko }\end{array}$ & $\begin{array}{l}4 \text { morts, } 1500 \text { sans-abris } \\
\text { et } 30000 \text { sinistrés }\end{array}$ \\
\hline
\end{tabular}

Tableau 1. Catastrophes naturelles historiques dans la ville de Douala. Sources : Bruckmann et al., (2019), Cameroon Tribune, Enquêtes de terrain.

le terrain (Figure 2), ce sont les quartiers les plus affectés par les catastrophes naturelles à Douala.

L'échantillonnage probabiliste en grappes à deux degrés est opté dans le cadre de cette recherche ; les grappes de la population cible correspondent aux zones à risque dans lesquelles les ménages à enquêter ont été identifiés, les questionnaires ont été administrés à un échantillon de ménage identifié de façon aléatoire à partir de la source du danger jusqu'à la zone hors danger. L'identification des ménages tient compte de plusieurs variables, pour une meilleure représentativité : âge moyen, genre, niveau d'étude, durée de résidence, statut d'occupation du logement, revenu moyen, statut professionnel (Tableau 2).

En complément d'analyse, 6 entretiens semidirectifs sont réalisés auprès des responsables desdits quartiers afin de mieux retracer l'inscription spatiale et temporelle des risques naturels et de leur gestion.

\section{PERCEPTIONS DES RISQUES NATURELS}

\section{A. Connaissance des risques naturels}

Dans l'analyse du questionnaire administré aux ménages cibles, il en ressort une grande conscience de l'exposition des enjeux aux aléas naturels (Figure 3a). Le sentiment d'exposition aux dangers naturels permet d'apprécier la mémoire que les populations exposées ont des risques naturels qu'elles côtoient quotidiennement. Le danger est permanent pendant la saison des pluies, mais différemment apprécié par les individus concernés, une forte majorité des ménages enquêtés juge être exposée aux aléas naturels $(87 \%)$. Cette exposition s'échelonne sur plusieurs degrés dont $43 \%$ des ménages enquêtés se sentent moyennement exposés et $8 \%$ fortement exposés (Figure $3 b$ ).

Les inondations sont considérées comme le risque naturel le plus présent dans les quartiers concernés (67\%), tandis que les mouvements de terrain sont moins présents (Figure 3c). Deux aspects peuvent expliquer cette situation : la configuration du relief favorise une colonisation accrue des vallées inondables, des espaces à mangrove et des zones marécageuses ; les pentes instables représentent une mineure partie de l'espace urbanisé. L'urbanisation s'est prioritairement effectuée dans les zones basses, les plateaux situés à l'arrière-plan du bassin sédimentaire ont les marques récentes d'urbanisation ; les collines de la Nsape situées aux limites de la ville de Douala sont actuellement en cours d'urbanisation.

La connaissance des causes des catastrophes naturelles constitue un axe majeur d'évaluation de la perception, elle permet d'apprécier le niveau de connaissance des mécanismes qui entourent la manifestation des risques naturels (Figure 3d). L'arrivée des pluies est considérée comme l'élément majeur dans le déclenchement des catastrophes naturelles $(62 \%)$, certains ménages invoquent les idées surnaturelles en percevant les catastrophes naturelles comme une manifestation de la punition divine (2\%). D'autres facteurs tels les changements climatiques (17\%), l'urbanisation (11\%) et la fréquence des marées ( $8 \%$ ) sont évoqués comme causes des évènements naturels dommageables.

Les risques naturels influencent différemment la vie locale (Figure 3e), les ménages enquêtés se 


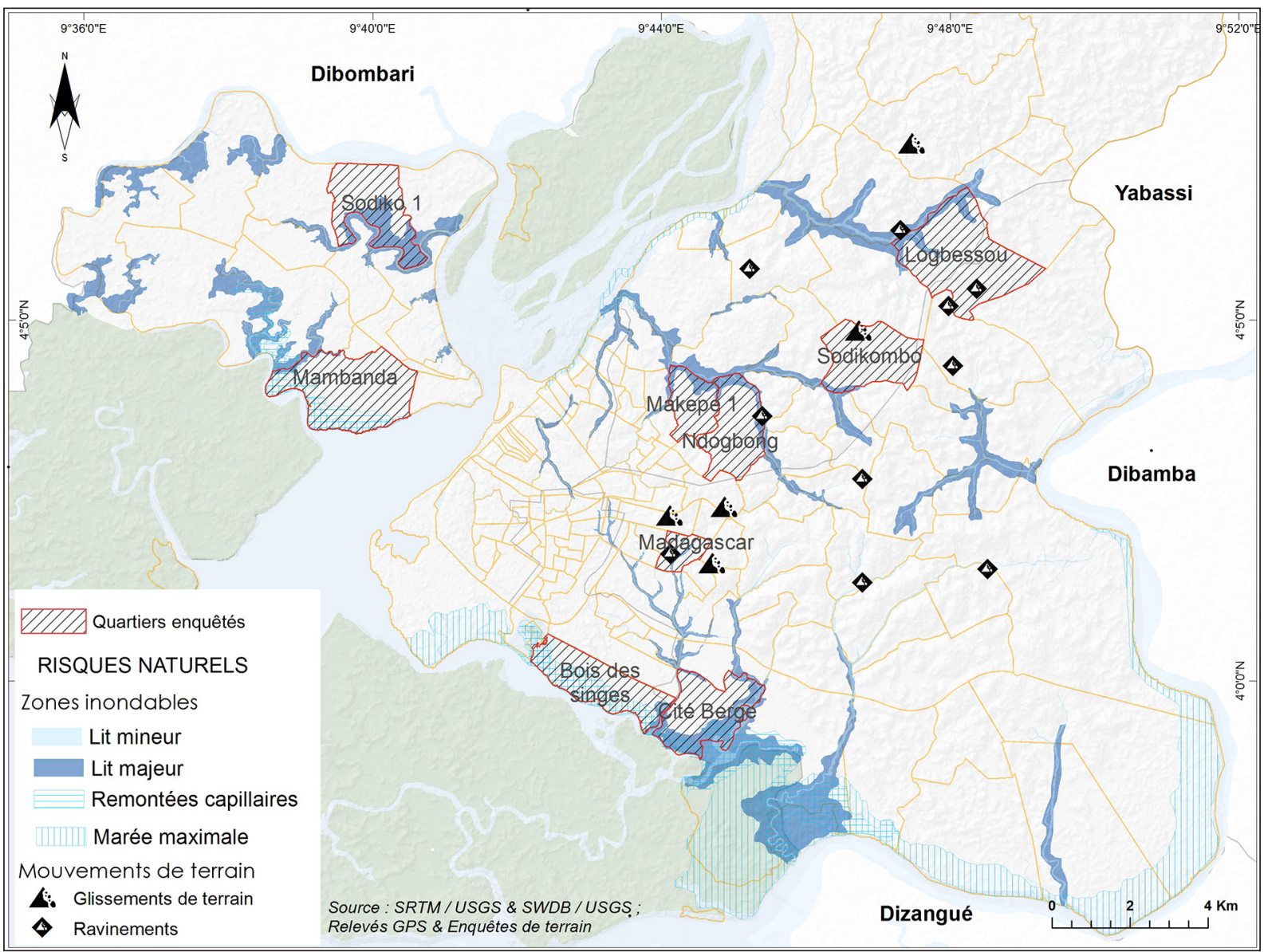

Figure 2. Localisation des quartiers enquêtés

\begin{tabular}{|l|l|l|l|}
\hline Variables & Indicateurs & Effectif & (\%) \\
\hline \multirow{4}{*}{$\begin{array}{l}\text { Âge } \\
\text { moyen }\end{array}$} & $20-34$ ans & 230 & 30 \\
\cline { 2 - 4 } & $35-49$ ans & 214 & 28 \\
\cline { 2 - 4 } & $50-64$ ans & 199 & 26 \\
\cline { 2 - 4 } & 65 ans et + & 122 & 16 \\
\hline \multirow{4}{*}{$\begin{array}{l}\text { Niveau } \\
\text { d'études }\end{array}$} & sans niveau & 69 & 9 \\
\cline { 2 - 4 } & primaire & 99 & 13 \\
\cline { 2 - 4 } & secondaire & 444 & 58 \\
\cline { 2 - 4 } & supérieur & 153 & 20 \\
\hline \multirow{4}{*}{$\begin{array}{l}\text { Durée de } \\
\text { résidence }\end{array}$} & 0 à 2 ans & 145 & 19 \\
\cline { 2 - 4 } & 3 à 5 ans & 260 & 34 \\
\cline { 2 - 4 } & 6 à 10 ans & 161 & 21 \\
\cline { 2 - 4 } & +10 ans & 199 & 26 \\
\hline
\end{tabular}

\begin{tabular}{|l|l|l|l|}
\hline Variables & Indicateurs & Effectif & $(\mathbf{\%})$ \\
\hline \multirow{4}{*}{ Genre } & masculin & 367 & 48 \\
\cline { 2 - 4 } & féminin & 398 & 52 \\
\hline \multirow{4}{*}{$\begin{array}{l}\text { Statut } \\
\text { professionnel }\end{array}$} & pauvre & 390 & 51 \\
\cline { 2 - 4 } & intermédiaire & 283 & 37 \\
\cline { 2 - 4 } & riche & 92 & 12 \\
\hline \multirow{3}{*}{$\begin{array}{l}\text { Statut d'occupa- } \\
\text { tion du logement }\end{array}$} & public & 99 & 13 \\
\cline { 2 - 4 } & privé formel & 138 & 18 \\
\cline { 2 - 4 } & privé informel & 474 & 62 \\
\cline { 2 - 4 } & chômeur/inactif & 54 & 7 \\
\hline & locataire & 283 & 37 \\
\hline
\end{tabular}

Tableau 2. Caractéristiques des ménages enquêtés

sentent majoritairement en danger face aux menaces naturelles $(71 \%)$, tandis qu'une minorité juge être en sécurité dans les sites potentiellement dangereux (29\%). Pour ce qui est des degrés de conscience du risque (Figure 3f), $17 \%$ des ménages enquêtés a une forte conscience du risque, tandis que $13 \%$ considère les dangers naturels négligeables. Au-delà de ces extrêmes, une frange importante des ménages considère les risques naturels comme un danger permanent (36\%), tandis que $34 \%$ les perçoit comme un évènement inoffensif. 
dans les pays en développement. L'exemple de Douala (Cameroun)

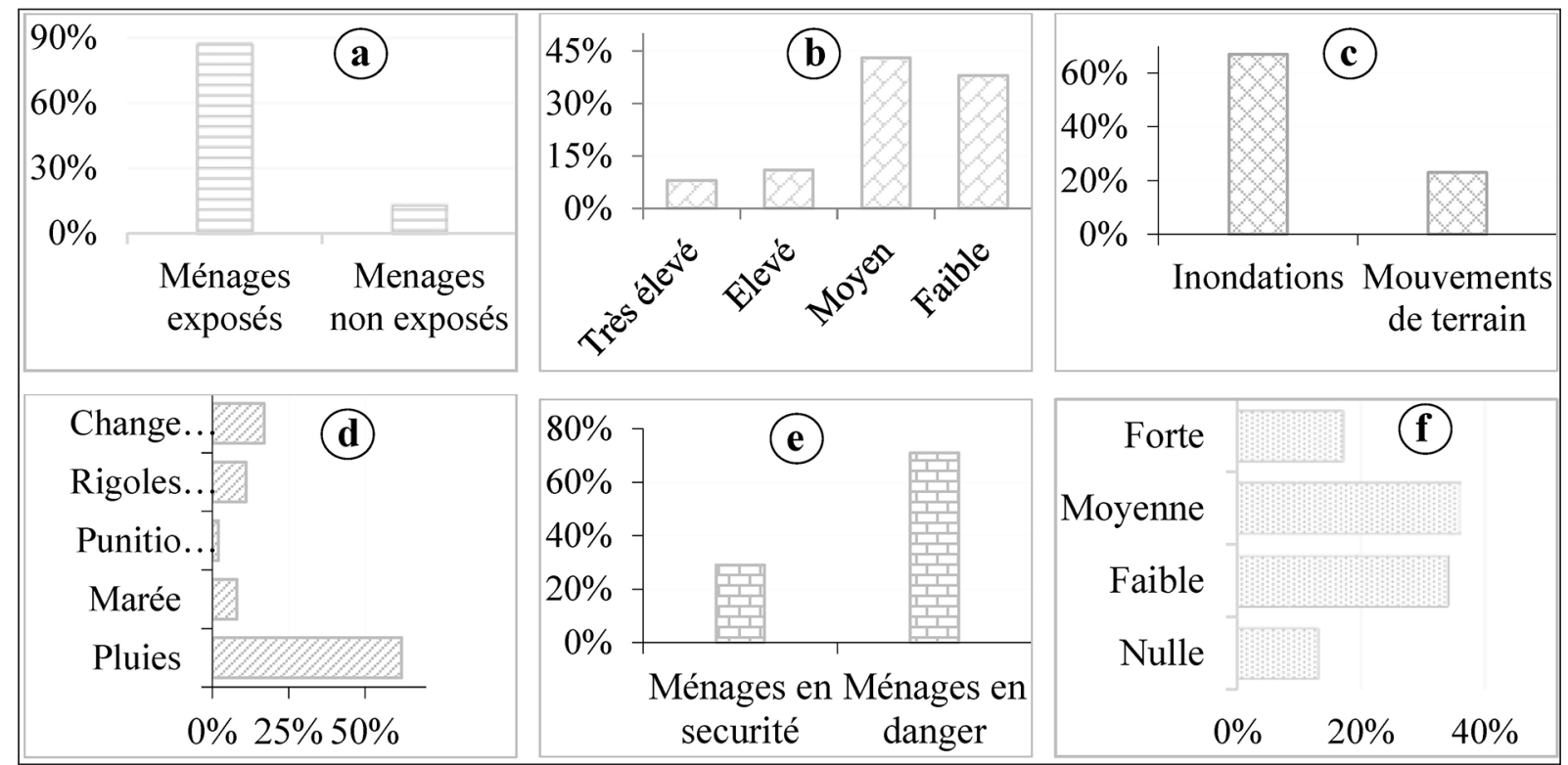

Figure 3. Perception des risques naturels. La Figure 3a relève le sentiment d'exposition des ménages aux aléas naturels, les ménages exposés apprécient différemment leur niveau d'exposition ( $3 b)$ et sont davantage menacées par les inondations (3c). La Figure 3d évalue le niveau de connaissance des causes de la menace naturelle, les Figures $3 \mathrm{e}$ et $3 \mathrm{f}$ analysent respectivement le sentiment de sécurité et les degrés de conscience du risque.

Une autre analyse se penche sur le rôle de l'âge dans les représentations des risques naturels, les personnes âgées minimisent moins les menaces naturelles et les acceptent plus facilement que les personnes moins âgées. Dans la classe [Élevé Fort], les âges les plus représentés sont supérieurs à 45 ans. Une valeur de $5 \%$ des plus de 65 ans se représente la menace naturelle comme un danger fort ; contre $2 \%$ des [45-65 ans], $1 \%$ des [25-45 ans] et une faible considération chez les moins de 25 ans. En croisant ces données avec comme variable de contrôle la durée de résidence dans le quartier, de nouvelles tendances se dessinent tout en renforçant cette hypothèse.

La conscience des risques naturels est influencée par un ensemble de facteurs socio-économiques, elle est analysée ici en fonction du genre, du revenu et de la durée de résidence dans le quartier (Figure 4). L'analyse basée sur le genre affiche $10 \%$ de femmes et $7 \%$ des hommes qui ont une forte conscience des risques naturels, $20 \%$ d'hommes et $14 \%$ de femmes considèrent les risques naturels comme un phénomène insignifiant. La durée de résidence dans le quartier influence aussi la conscience du risque (Figure $3 \mathrm{~b}$ ), les ménages ayant plus de 06 ans dans le site étudié ont une grande conscience du risque (15\%), les ménages nouvellement installés ( $<5$ ans) ont une faible conscience des risques naturels $(6 \%)$.
La corrélation entre la durée de résidence et la perception du sentiment de sécurité par les ménages exposés relève une valeur élevée ( $28 \%$ ) pour les résidents de plus de 10 ans (Figure 4e). Le genre féminin se considère en danger dans les sites à risque (40\%), contrairement au genre masculin (31\%); le sentiment de sécurité est quasiment développé chez les femmes (Figure 4b). Les femmes sont davantage intéressées aux problématiques environnementales à cause de leur proximité avec la nature, le travail des femmes dépend étroitement de la nature pour satisfaire leur besoin de subsistance, elles sont majoritairement impliquées dans le secteur privé informel (69\%).

Comme en témoignent les recherches effectuées en Équateur (D'Ercole, 1996) ou au Niger (Maichanou, 2014), la perception des risques naturels varie avec le revenu des ménages. Les ménages pauvres ont une faible évaluation de la menace, ils se considèrent en sécurité malgré les dommages enregistrés lors des catastrophes précédentes (Figure $4 d$ ). Les ménages pauvres ont une perception différenciée des risques par rapport aux ménages riches, ils ont une faible conscience des risques naturels contrairement aux ménages riches (Figure $4 \mathrm{c}$ ). Comme l'expliquent plusieurs études (Lechowska, 2018 ; Verlynde, 2018 ; Burningham et al., 2008 ; Gollier, 2005), il existerait une relation positive entre les inégalités de revenus et la 


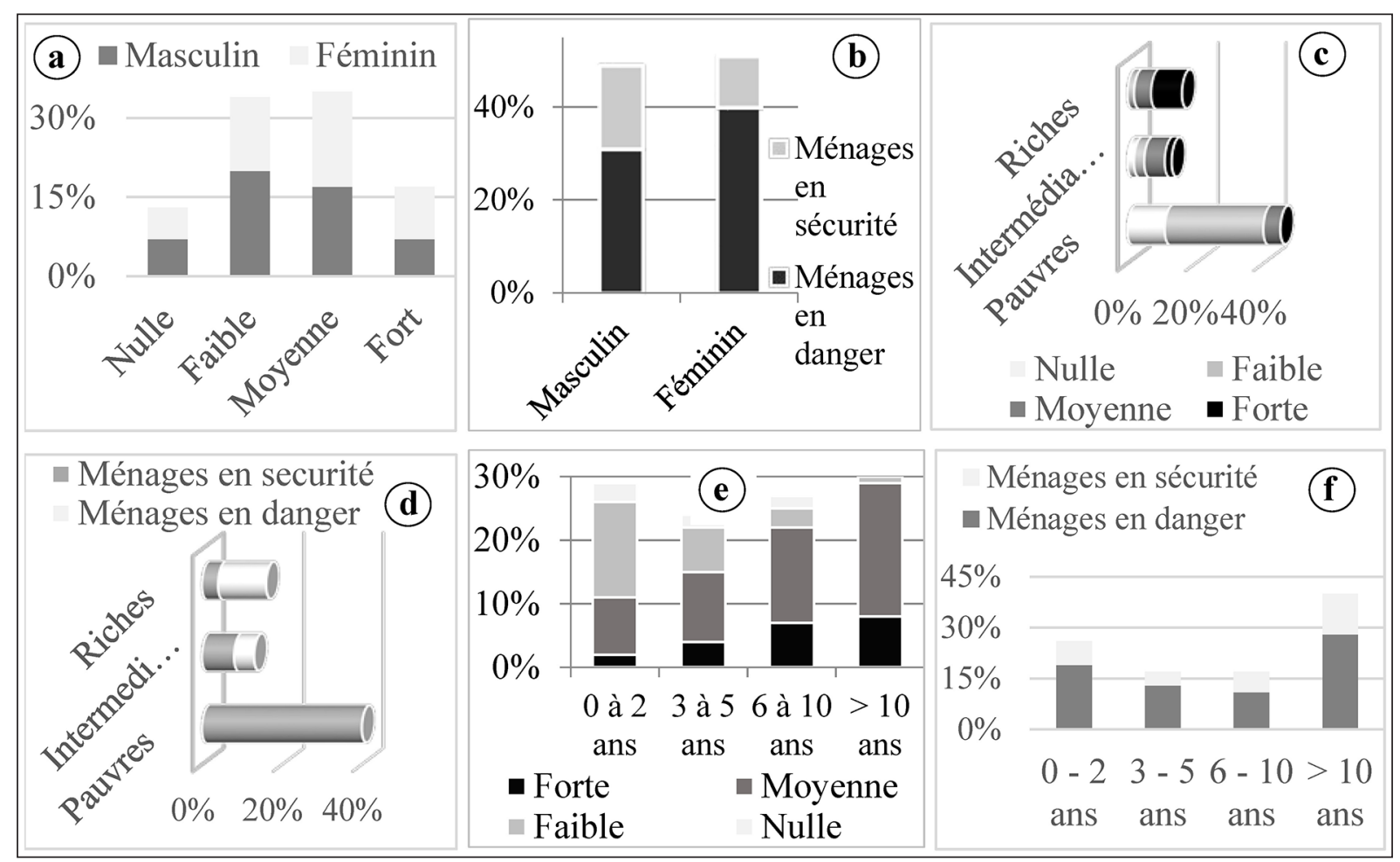

Figure 4. Perception du risque en fonction des facteurs socio-économiques. Les degrés de conscience sont fonction du genre (4a), de la durée de résidence (4e) et du revenu (4c). Le sentiment de sécurité est aussi fonction du revenu (4d), du genre (4b), de la durée de résidence (4f)

perception des risques, les ménages aux revenus les plus bas ont tendance à ignorer ou à minimiser le risque (Armas et al., 2015).

L'analyse de la perception en fonction de la distance à la menace produit des niveaux hiérarchiques de perception à partir de l'épicentre de l'aléa. Comme le souligne Crocq (1994), les comportements des enjeux humains exposés sont différents en fonction du zonage de la catastrophe, des réactions préventives sont enregistrées au fur et à mesure qu'on se rapproche de la zone d'impact (Figure 5). Deux secteurs sont mis à contribution pour analyser cette réalité, ils représentent les aléas d'inondation et de mouvements de terrain. Les ménages proches des cours d'eau ont un sentiment d'exposition aux inondations plus élevé par rapport à ceux situés en dehors de la zone d'impact; plus on se rapproche du lit du fleuve, plus la conscience du risque est élevée (Figure 5a). Dans le cas du ravinement de Ndogbong, les ménages situés dans la zone d'impact ont un sentiment de sécurité faible car les degrés de menace y sont élevés (Figure 5b), ces ménages ont pour la plupart déjà subi des dégâts lors des catastrophes passées (perte des parcelles, destruction des biens) et ont de ce fait développé une forte familiarité au risque.
La partie du questionnaire dédiée à la connaissance des risques naturels révèle que les ménages enquêtés ont une conscience accrue du danger qui les côtoie au quotidien, cette perception est fonction du genre, de la durée de résidence, du revenu des ménages et de la distance à la source du danger.

\section{B. Connaissance des moyens de protection et prévention des risques naturels}

La connaissance des moyens de protection et la prévention des risques naturels par les ménages exposés constituent un axe d'analyse prioritaire. Au-delà de la connaissance des risques naturels, la perception des moyens de protection peut améliorer la résilience des ménages exposés. Il n'est pas question ici d'un inventaire des moyens de protection des populations sur les sites concernés (espace vécu), il s'agit par contre d'une analyse de la perception qu'ont les ménages exposés sur les mesures de protection conduisant à la mitigation de l'impact des évènements naturels dommageables dans leur environnement (espace perçu).

Une question accorde une importance aux attitudes adoptées pendant la catastrophe naturelle, elle permet d'apprécier les possibilités d'actions pouvant 


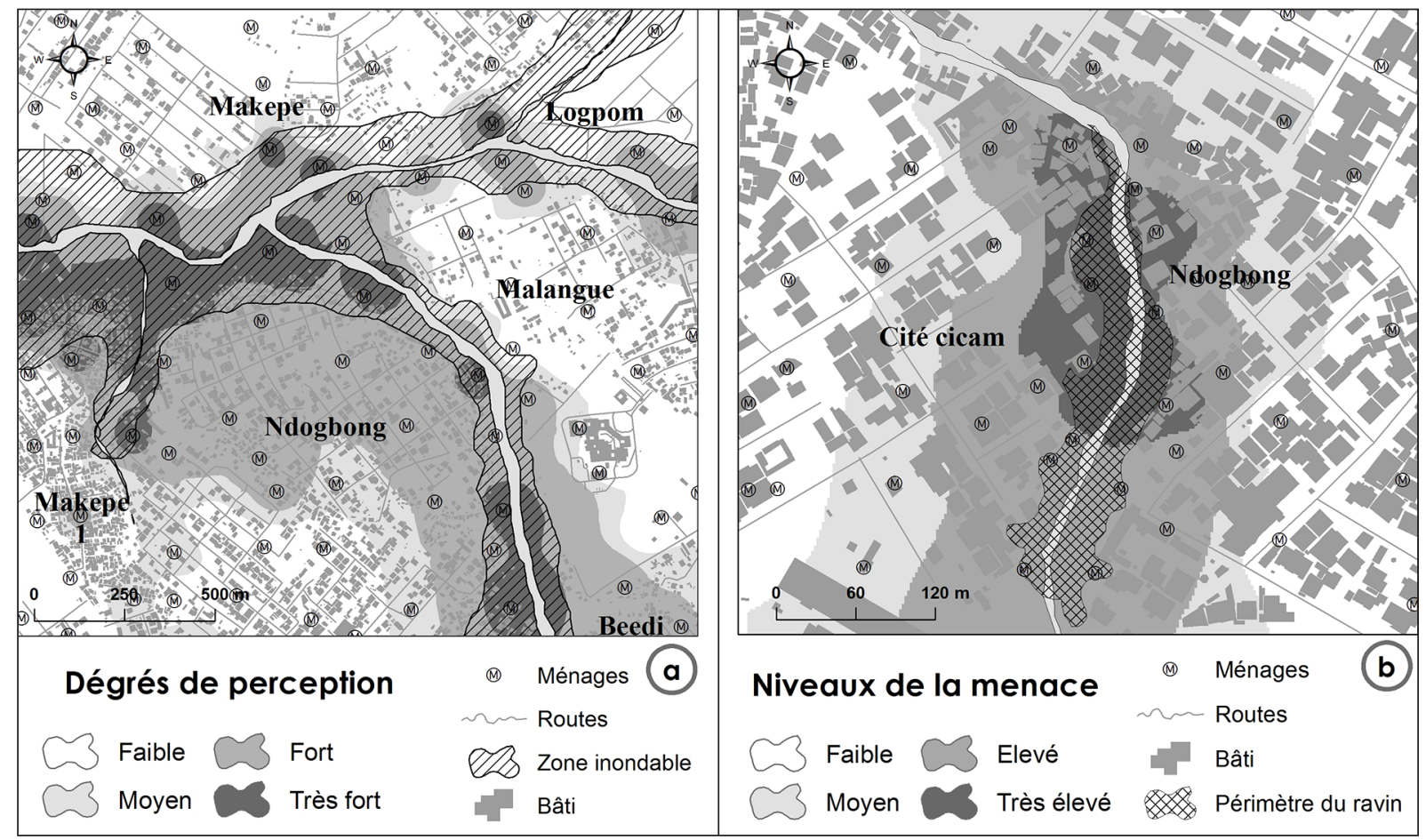

Figure 5. Perception des risques naturels en fonction de la distance à l'aléa. Distance par rapport au fleuve (5a), distance par rapport au ravin $(5 b)$

limiter l'endommagement. Le renforcement de la protection est majoritairement cité par les ménages exposés comme étant l'attitude à adopter s'il est annoncé une catastrophe imminente (Figure 6a). Dans les situations de catastrophe d'inondation les ménages perçoivent la fuite comme la dernière option (3\%); ils accordent une priorité successivement au renforcement de la protection (47\%), au renseignement (24\%), au confinement (17\%) et à la demande d'aide $(9 \%)$. Les ménages enquêtés misent plus sur l'adoption de comportements raisonnées (faire face au danger) au détriment des réactions instinctives (fuir, se réfugier ailleurs), ceci montre le degré d'accoutumance des ménages aux risques naturels.

Dans le scénario d'une inondation dommageable comparable à celle de la nuit du 03/04 août 2000 (référence locale), les ménages ont des perceptions différenciées sur les moyens de protection (Figure $6 b)$. Le curage des drains reste le moyen de protection le plus sollicité face à la lutte contre les inondations (64\%), les mesures relevées font majoritairement référence aux ouvrages hydrauliques, permettant ainsi une amélioration du système de drainage. La modalité construction sur pilotis représente $13 \%$ des observations, le déguerpissement des habitations installées dans le lit des cours d'eau est souhaité par $9 \%$ des ménages enquêtés, $8 \%$ des ménages sollicite par contre la mise sur pied des barrières de protection face aux inondations.

En matière de responsabilités dans la prévention des risques naturels (Figure 6c), les collectivités locales sont indexées comme étant les principaux acteurs, elles enregistrent une confiance de la part des ménages s'élevant à $74 \%$ (CUD, communes), au détriment de l'État central (12\%), les populations sont moins prises en compte (3\%). L'analyse de la perception des moyens de protection face aux risques naturels permet d'appréhender l'état de préparation des ménages en situation de crise, c'est une composante essentielle de la vulnérabilité des enjeux. Pour analyser les mesures préventives avant la catastrophe, les ménages ont été appelé à répondre sur un présupposé ordre d'évacuation en cas d'alerte ; les données obtenues sont couplées avec les revenus des ménages (Figure 6d). Les ménages pauvres ${ }^{1}(61 \%)$ ont une faible perception de l'alerte et refusent d'évacuer malgré la gravité potentielle de l'évènement; les ménages riches (11\%), sont prêts à évacuer en cas d'alerte d'un évènement naturel dommageable. La résistance à l'évacuation trouve son fondement dans la difficulté pour les ménages d'avoir accès à un nouveau logement, le sentiment de sécurité qui anime certains et l'opportunité économique dans le site occupé. 


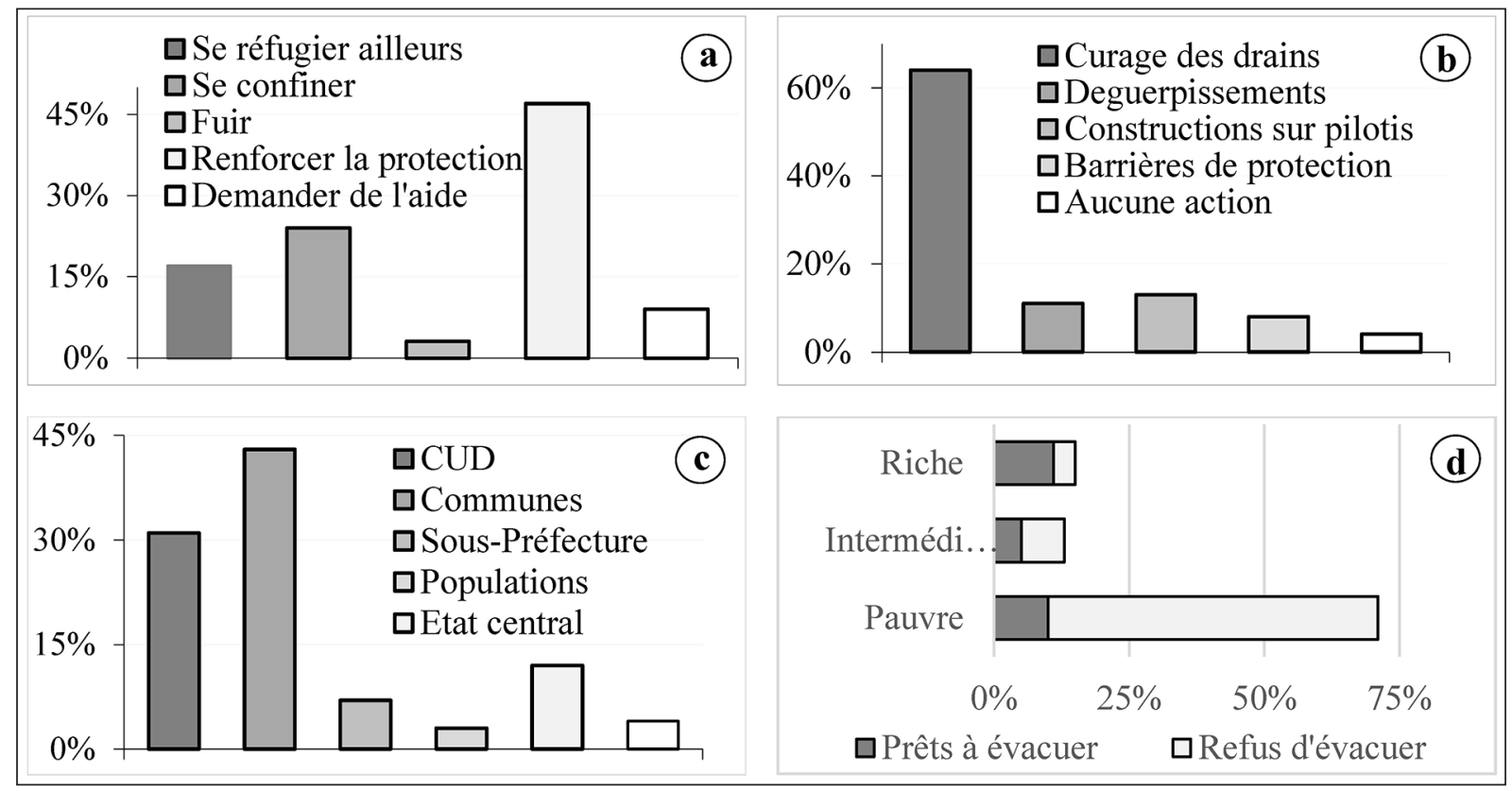

Figure 6. Perception de la prévention en situation de risque naturel. Attitudes probables en situation de catastrophe (6a), connaissance des moyens de protection (6b), Attribution des responsabilités (6c), scenario d'évacuation et statut économique $(6 \mathrm{~d})$

\section{Informations sur les risques naturels}

La connaissance des mesures de protection et la prévention face aux risques naturels dépendent de la qualité des informations reçues. Les informations préventives sur les risques naturels font l'objet d'une perception différenciée par les ménages, le faible taux de réponses en faveur de la communication sur les risques ne met pas en doute les activités développées par les acteurs publics locaux dans ce sens, cela est lié au faible taux de pénétration des informations dans les zones à risque (Olinga, 2012 ; Mounde, 2019). À la question de savoir si les ménages ont reçu les informations sur la dangerosité du site, une majorité juge n'avoir jamais été informé officiellement sur les risques naturels avant leur implantation, certains ont été informé une fois installés sur le site.

En analysant la perception des moyens de sensibilisation présents dans les sites à risque, quatre modalités synthétisent les avis des ménages (Figure 6a). Les ménages estiment majoritairement avoir pris connaissance des risques naturels une fois sur le terrain $(51 \%)$, cette perception est partagée entre ceux ayant constaté par observation du site et ceux ayant compris la dangerosité du site après la première expérience d'une catastrophe naturelle. L'information orale reste le deuxième canal d'information sur les risques menaçant les localités du littoral camerounais, elle représente $44 \%$ des modalités proposées. Pour ce qui concerne les ménages propriétaires de terrain, l'achat des parcelles de terrain a été précédé par un renseignement sur les caractéristiques du quartier et du site, c'est à travers cette règle d'usage qu'ils prennent connaissance de la dangerosité du site. L'interrogation qui se dégage est de savoir les motivations d'achat d'un terrain situé en zone à risque, ayant connaissance de la fréquence de l'évènement naturel.

Dans certains sites dangereux, des pancartes sont installées pour interdire les constructions, cette tâche est effectuée par la communauté urbaine de Douala, $4 \%$ des ménages enquêtés mentionne cet outil comme marqueur des territoires dangereux (Figure 7b). Le dernier outil d'information sur les territoires à risque naturel est le dépliant (1\%), il est régulièrement distribué dans les campagnes de sensibilisation mais à un public ciblé.

L'information préventive sur les risques naturels atteint peu les populations concernées, le Tableau 3 est un résumé des canaux de communication préventive sur les risques naturels d'après les ménages enquêtés. La radio est perçue comme le moyen souhaité pour la transmission des informations préventives sur les risques naturels (52\% - choix 1 et $48 \%$ - choix 2), par ce moyen les populations prendront majoritairement acte des informations 


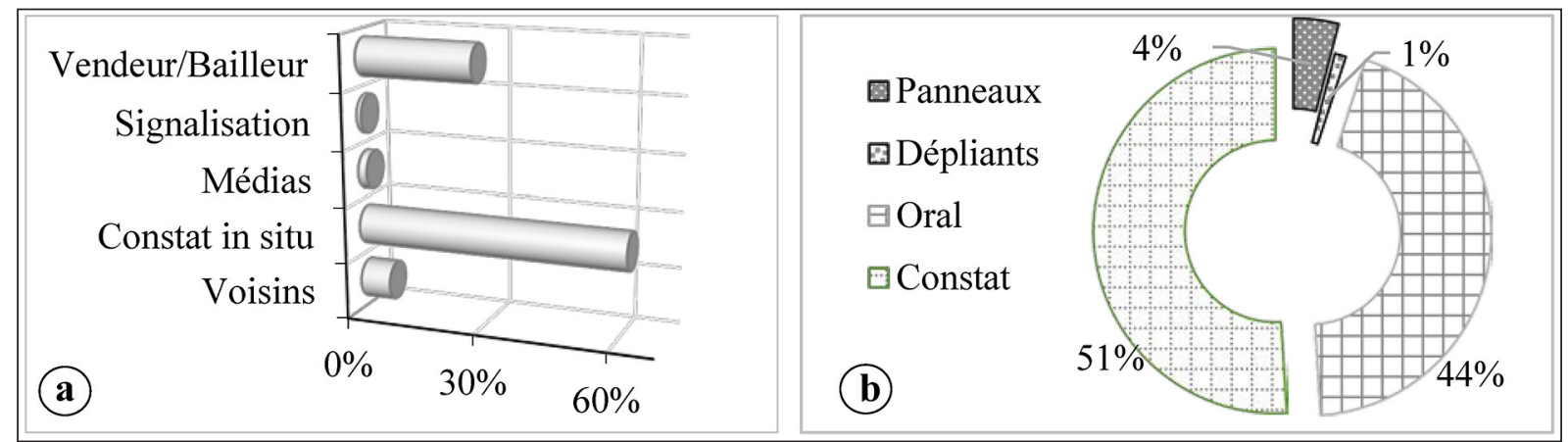

Figure 7. Informations sur les sites à risque. Informations sur l'état du site avant l'occupation (7a), perception des canaux d'information (7b)

\begin{tabular}{|l|l|l|l|l|l|}
\hline \multirow{2}{*}{$\begin{array}{l}\text { Canaux } \\
\text { d'information }\end{array}$} & \multicolumn{6}{|l|}{ Fréquences du choix ordonné des ménages (en \%) } \\
\cline { 2 - 7 } & choix $\mathbf{1}$ & choix 2 & choix 3 & choix 4 & choix 5 \\
\hline Télévision & 19 & 24 & 17 & 2 & 15 \\
\hline Radio & 52 & 48 & 22 & 9 & 1 \\
\hline SMS & 12 & 10 & 28 & 31 & 16 \\
\hline Internet & 4 & 7 & 9 & 47 & 59 \\
\hline Presse écrite & 13 & 11 & 24 & 11 & 9 \\
\hline
\end{tabular}

Tableau 3. Canaux d'information préventive préférés par les ménages

transmises, la diversité des radios locales joue un rôle primordial dans le relais des informations. En fonction des priorités, la télévision (19\% - choix 1 et $24 \%$ - choix 2 ), la presse écrite ( $13 \%$ - choix 1 et $11 \%$ - choix 2) et la communication par SMS via le signal GSM (12\% - choix 1 et $10 \%$ - choix 2) sont considérés moins importants que la radio. Les ménages enquêtés suggèrent aussi la communication par internet comme dernier recours pour relayer l'information sur les risques naturels ( $47 \%$ - choix 4 et $59 \%$ - choix 5).

L'information préventive sur les risques naturels permet de sensibiliser la population sur les dangers encourus dans les sites concernés, elle renforce la culture du risque et oriente les constructions mentales. C'est un élément à prioriser dans les politiques de gestion non-structurelles des risques naturels.

\section{DES CONSTRUCTIONS MENTALES AUX COMPORTEMENTS SOCIAUX}

\section{A. Représentations sociales des risques naturels}

Dans l'acte perceptif, l'individu exposé se fait une idée de la menace afin de décider sur les attitudes à adopter par la suite. Cette représentation des informations dédiées aux évènements naturels contribue à expliquer les comportements qui en découlent. Dans un territoire donné, les individus sont soumis aux menaces naturelles d'une intensité relative à leur distance par rapport à la source du danger. Les représentations des risques naturels sont ainsi différentes d'un individu à l'autre, même au sein de communautés exposées aux menaces naturelles de même intensité. Les constructions mentales réconfortent l'individu dans sa proximité avec la menace naturelle et permettent de trouver une responsabilité externe, en d'autres termes elles aident l'individu à justifier son installation dans la zone.

Les risques naturels sont tolérés par les ménages exposés, c'est le résultat de l'analyse des variables résumant le questionnaire administré aux ménages et mettant en exergue les représentations sociales. Les ménages enquêtés jugent avoir une bonne conscience des risques naturels $(81 \%)$ qu'ils côtoient au quotidien, ils se sentent en sécurité (71\%) malgré la forte exposition aux menaces naturelles (87\%). Le niveau de sévérité des catastrophes naturelles est relatif, leur représentation en fait un évènement naturel peu craint par les populations locales $(21 \%)$. Les variables liées à la prévention et à l'information préventive évaluent la capacité des populations à les intégrer comme outils pratiques de gestion des risques naturels; globalement, les 
ménages sont moins informés et tant bien même que cette information leur soit transmisse efficacement, les représentations qu'ils se font des risques naturels changent peu à cause des facteurs socio-économiques et des paramètres de l'aléa.

Les inondations et les mouvements de terrain sont représentés dans la ville de Douala comme un évènement intrinsèque au territoire, les ménages concernés les ont progressivement intégrés dans leur vécu quotidien, ces risques naturels ont été domestiqués et une véritable manufacture des territoires à risque se développe dans les quartiers précaires. Cette simplification des risques naturels par les ménages traduit leur degré d'acceptabilité. Dans leur procédé de minimalisation des risques naturels, les ménages relativisent l'impact des dégâts occasionnés en le comparant à ceux constatés chez les voisins ou avec la situation des autres quartiers.

Au-delà de la minimisation des risques naturels, une autre tendance des ménages migre totalement vers le déni de ces risques et considère ces évènements comme des faits normaux, se produisant à des périodicités précises. La passivité observée s'explique par l'impact peu traumatique des catastrophes naturelles passées d'une part, et d'autre part par des facteurs économiques qui limitent les capacités des populations à adopter un comportement préventif (remblais, reboisement, mur de protection, barrières de sécurité, etc.). Cette impuissance face aux menaces naturelles modélise les représentations sociales, les populations au capital financier ou social faible acceptent les risques naturels tant que cela ne cause pas des pertes en vies humaines, malgré le fort impact enregistré sur le capital physique (destruction des biens matériels). Une menace sur la vie des populations exposées conduit à une crainte élevée de l'évènement dommageable en question.

Les représentations sociales sont fonction d'un ensemble d'éléments qui constituent l'individu ou le groupe social. Les variables socio-démographiques (âge, genre, niveau d'éducation) et économiques (revenu) sont quelques facteurs pouvant déformer les idées que les populations ont des risques naturels. Les risques sont différemment présents dans les esprits, $20 \%$ de femmes et $16 \%$ d'hommes représentent les risques naturels comme un évènement insignifiant. Par ailleurs, $18 \%$ d'hommes et $25 \%$ de femmes considèrent les risques naturels comme un danger moyen.
Les classes sociales n'ont pas un même regard sur les faits de société, encore moins sur les problématiques environnementales, car les contacts avec la nature ne sont pas identiques et surtout les capacités à faire face aux évènements naturels dommageables sont variables. Les ménages pauvres se représentent moins exposés aux dangers naturels contrairement aux ménages riches qui s'expriment plus dans les degrés de danger «Élevé » et " Très élevé ». Les ménages pauvres, impuissants face aux risques naturels, se cantonnent majoritairement dans la simplification de la menace naturelle afin de montrer leur maitrise parfaite de la situation. Le facteur économique tout en jouant un rôle de handicap ou de force en situation de risque naturel, modifie les représentations sociales.

\section{B. Attitudes sociales en situation de risque naturel}

Les variables intervenant dans l'évaluation des capacités des ménages à agir efficacement en situation de risque naturel concernent le capital physique (biens matériels), le capital social (éducation, santé, taille des ménages, assainissement, accessibilité, genre) et le capital économique (revenu). Les seuils de variables sont définis par la valeur 0 ou 1 , qui traduit la présence ou l'absence de l'élément. Par pondération et agrégation des valeurs obtenues à l'échelle de chaque ménage, on obtient des indices de bien-être qui caractérisent de nombreuses privations en situation de risque naturel. Les ménages en deçà du seuil de bien-être (0.5) vivent dans des conditions jugées précaires (absence de drainage, mauvaise gestion des déchets, faible accessibilité, faible revenu, soins de santé à domicile, sous nutrition ou malnutrition, faiblesse du capital social, famille nombreuse, précarité de l'emploi, absence de protection sociale). Cette situation complique davantage la survie des ménages pauvres d'où leur faible résilience.

À partir de cette grille d'analyse, $71 \%$ des ménages enquêtés sont dans une situation de précarité sociale, cela traduit leur faible niveau de bienêtre d'où leur forte vulnérabilité face aux risques naturels. Ces ménages peinent à satisfaire leurs besoins vitaux, d'où leur difficulté à se protéger efficacement contre les risques naturels. Par contre, les ménages possédant assez de potentialités, peuvent facilement les convertir en actions sociales préventives, ils sont moins vulnérables aux risques naturels (29\% des ménages). Le niveau de bien- 
dans les pays en développement. L'exemple de Douala (Cameroun)

être des ménages dans les sites exposés est relatif, mais une majorité (56\%) s'estime insatisfait des conditions de vie. Une perception étonnante pour un individu extérieur au territoire concerné, qui y voit plutôt des ménages dans une précarité et impuissants face aux dangers naturels. Pour appuyer l'analyse sur le bien être des ménages exposés, une question sur un scenario possible de recasement dans un site plus sécurisé leur est posée. Plus de $2 / 3$ des ménages ( $73 \%$ ) est prêt à s'installer ailleurs s'il leur était demandé, pourvu que les charges ne soient pas à leur compte. Les ménages ne désirant pas s'installer ailleurs s'il leur était proposé tirent un quelconque bénéfice de leur habitation en zone à risque (commerce, agriculture).

$\mathrm{Au}$ demeurant, le niveau de bien-être influence les comportements des ménages en situation de risque naturel, l'adoption des actions préventives est corrélée à une vulnérabilité [Moyen à Élevé], les ménages en situation de précarité sociale font face à des privations matérielles limitant les actions préventives. Dans la gestion collective des risques naturels, la cohésion sociale permet d'activer le levier de la résilience collective, l'appartenance des ménages aux associations de développement à l'échelle du quartier ou du bloc constitue un apport indispensable dans le retour à la normale après un évènement naturel dommageable. Ce capital social est à prendre en compte dans l'analyse du bien-être de l'individu en situation de risque naturel, $74 \%$ des ménages juge leur efficacité lors de la phase de reconstruction. Face à la menace naturelle, plusieurs attitudes sociales sont enregistrées, ces comportements dépendent de la perception et des représentations sociales, l'adoption des comportements par les ménages est motivée par les paramètres de l'aléa naturel et surtout les possibilités d'actions qui s'offrent à eux pendant la gestion de crise (Figure 8).

De l'image perçue aux attitudes sociales, un faisceau d'éléments contextuels façonne les comportements des individus en situation de risque naturel. Les réactions des ménages dépendent d'une part des caractéristiques de l'aléa et d'autre part des facteurs socio-économiques qui caractérisent la vulnérabilité sociale. Pour mettre en relation le capital financier des ménages et l'adoption des comportements préventifs, nous avons fait appel au test de Khi 2. L'hypothèse défendue stipule que les capacités d'action des ménages conditionnent l'adoption des mesures protectrices. Le résultat obtenu montre que $35 \%$ de ménages pauvres, $64 \%$ de ménages intermédiaires et $89 \%$ de ménages riches penchent en faveur de mesures protectrices; tandis que $65 \%$ de ménages pauvres, $36 \%$ de ménages intermédiaires et $11 \%$ de ménages riches développent des attitudes non protectrices. Pour une valeur de Khi 2 calculé égale à 109,448 avec un ddl $=2$ et $p<0.0001$; il existe une relation entre le capital financier des ménages et l'adoption des mesures préventives. Ces comportements préventifs sont liés aux questions sur un ordre d'évacuation des lieux recommandé par les pouvoirs publics.

\section{OPTIMISATION DES COMPORTEMENTS PRÉVENTIFS}

La gestion des risques naturels à Douala est toujours tournée vers le tout-structurel, c'est le cas du drainage des drains et de la stabilisation des ravins instables. Cependant la communication semble toujours manquer d'efficacité dans ces projets urbains ce qui limite la résilience des ménages exposés. Néanmoins, les comportements préventifs peuvent être optimisés par une meilleure communication et surtout l'implication des ménages exposés dans le choix des politiques urbaines.

\section{A. Renforcement de la culture du risque}

La ville de Douala s'est récemment tournée vers la construction d'une ville durable en développant des projets d'aménagement à long terme (Olinga, 2012 ; Bruckmann et al., 2019), cette politique urbaine s'est soldée par la mise sur pied des plans locaux d'urbanisme intégrant les inondations dans la planification urbaine et les projets d'aménagement urbain dans les zones à risque (projet éboulement et projet ville durable). Ces projets ont conduit selon les responsables des quartiers, à la régression de la zone d'impact de l'aléa d'une part, et d'autre part à une réduction des mesures de prévention d'où la baisse de la conscience collective. La communication reste peu efficace dans les politiques de gestion des risques, les actions de prévention/information des populations se limitent aux représentants des quartiers et des chefferies traditionnelles, même les documents d'urbanisme n'ont pas fait l'objet d'une véritable politique d'information auprès des ménages (Meva'a et al., 2010 ; Mounde, 2019).

Malgré ces aménagements techniques, les catastrophes naturelles sont récurrentes et causent 


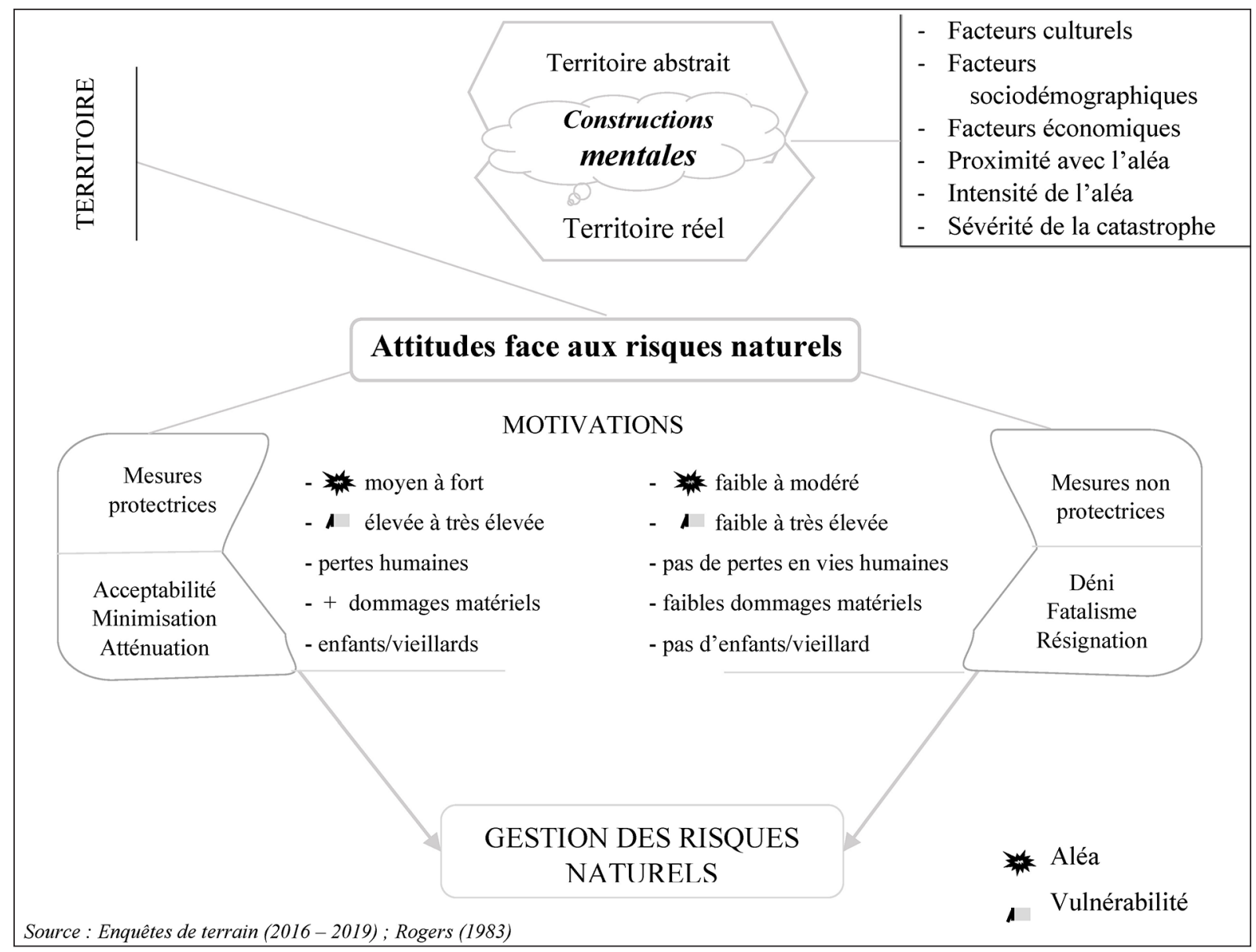

Figure 8. De l'information perçue aux attitudes sociales en situation de risque

davantage des dommages aux ménages exposés, les mesures effectuées sont jugées inadéquates par les responsables de quartiers car s'éloignant parfois des aspirations locales, cette réalité de l'échec des politiques de gestion structurelle des risques naturels est relayée par Betart et Fort (2014) dans les pays du Sud. Les ouvrages de drainage effectués dans le projet «Douala ville durable », au-delà de leur faible capacité à limiter les inondations dans la ville, constituent un autre risque pour les enfants car les outils de protection des berges sont inefficaces (risque de noyade - un décès en juin 2020). La connaissance des politiques locales par les ménages est un atout dans la gestion des risques (Olinga, 2012), elle conditionne la participation des populations exposées ; ces dernières se sentant impliquées, amplifient la portée des politiques locales de gestion des risques. Sur la base des ménages enquêtés, 83 $\%$ n'a pas connaissance de l'existence des actions publiques locales en faveur de la prévention des risques naturels à Douala. Une meilleure résilience des ménages passe par la vulgarisation des projets urbains dans les zones les plus vulnérables, les plans de prévention des risques développés en Martinique s'inscrivant dans cette dynamique ont montré leur efficacité (Léone, 2007).

À partir de l'analyse des résultats de l'enquête, il est indispensable de procéder à une réorientation de la communication et à la diffusion de l'information dédiée aux évènements naturels dommageables auprès des ménages exposés. Dans ce cadre, les ménages préfèrent la radio comme canal d'information préventive sur les risques naturels (voir Tableau 3), les posters et banderoles affichées par les autorités publiques sont jugées moins efficaces. Ainsi, la représentation des risques naturels par les ménages reste erronée, cela est lié à la perception de leur environnement immédiat; comme le souligne Chouari (2019), pour le cas de la Tunisie, l'amélioration des informations sur les risques permet de développer la culture de prévention et la conscience du risque. Dans ce cadre, les radios locales sont les meilleurs relais d'information préventive en faveur des territoires exposés.

La sensibilisation par la matérialisation des catastrophes passées (repères de crue, panneaux d'alerte/ 
dans les pays en développement. L'exemple de Douala (Cameroun)

danger) et la cartographie à petite échelle améliorent la compréhension du phénomène et renforcent de ce fait la mémoire collective (Thouret, 2003). L'exemple des risques générés par le drainage de la ville relève l'urgence d'une communication préventive, la signalisation de la dangerosité des cours d'eau en période de crue peut permettre d'éviter des dommages corporels. La sensibilisation doit prendre en compte les mesures de bonne pratique à adopter en cas de risque naturel, activités à développer dans les écoles et à intégrer dans les associations de développement au sein des quartiers exposés. Sans toutefois négliger la gestion structurelle, la réglementation dans les territoires à risque doit être accompagnée de mesures complémentaires permettant le renforcement de la culture du risque ; à l'exemple des plans de préparation et de prévention, des mesures d'alerte et de gestion de crise.

Une valorisation de l'information dédiée aux risques naturels auprès d'un public plus grand passe aussi par une cartographie interactive, elle n'exclut pas les formes traditionnelles de communication, car des centres d'intérêt peuvent être extraites de la plateforme et placés comme des posters dans les sites exposés. Le renforcement de la culture du risque par la diversification de la communication permet une appropriation des informations par les ménages exposés et l'adoption des comportements préventifs.

\section{B. L'amélioration de la résilience des ménages et du territoire}

L'ONU a encouragé la prise en compte de la résilience pour l'amélioration de la gestion des crises lors de la décennie internationale pour la réduction des catastrophes (Dauphine et Provitolio, 2007). Les recherches actuelles insistent sur la nécessité de créer des villes résilientes, afin de favoriser la durabilité des espaces et des sociétés (Boudières, 2007). Au-delà de l'amélioration des informations sur le risque comme élément de renforcement de la résilience sociale (Bercera et al., 2013), il est nécessaire d'impliquer les ménages concernés afin d'améliorer la portée des politiques locales (Mounde, 2019 ; Bruckmann et al., 2019). Les entretiens avec les responsables de quartiers révèlent que la non implication des populations locales dans les politiques urbaines reste une des causes du dysfonctionnement observé dans la gestion des risque naturels à Douala, les ménages exposés sont exclus des projets touchant leur environnement immédiat ; une meilleure implication des ménages va concourir à renforcer la portée des politiques urbaines axées sur la réduction des risques naturels. Le niveau de confiance dans les autorités locales (Figure 5c) constitue un atout majeur dans la définition des politiques locales plus intégrées au détriment de la gestion basé sur le tout structurel défini par l'État central.

Les analyses mises en exergue par Meva'a et al. (2010) sur la constitution des Conseils de Bassins versants pour la gestion des inondations sont une initiative pouvant davantage impliquer les riverains dans la bonne marche des politiques de réduction des risques naturels. Bétard et Fort (2014) relèvent que les risques sont différemment gérés entre les pays développés et ceux du Sud, ils encouragent la gestion des risques axée sur les variables de la vulnérabilité sociale qui s'assimile beaucoup plus à la notion de résilience. Renforcer la résilience des ménages passe d'une part par leur implication dans les politiques locales et l'amélioration de leur bien-être, le faire c'est contribuer à la réduction de l'impact des catastrophes naturelles, renforcer la prévention et améliorer la gestion de crise (Mounde, 2019). Le renforcement de la résilience sociale dans les territoires à risque naturel passe aussi par la réduction des facteurs conduisant à des actions à risque, il est nécessaire de développer des moyens d'alerte et de prévention pouvant contribuer à l'adoption des comportements préventifs.

Les catastrophes naturelles affectent beaucoup plus le bien-être des ménages, la faible capacité des ménages pauvres conduit à des dommages matériels importants et renforce de plus leur situation de précarité sociale (Hallegate et al., 2017). Afin de promouvoir des comportements préventifs et une meilleure résilience des ménages face aux risques naturels, il est important de contribuer à la réduction des vulnérabilités sociales à travers des outils permettant aux ménages de sortir de la pauvreté afin d'adopter des bonnes pratiques en situation de risque naturel. D'autre part, la durabilité des territoires à risque passe par l'intégration de ces risques dans les projets d'aménagement urbain, dans le but de maitriser et de sécuriser les implantations d'enjeux futurs. L'évaluation des comportements sociaux en situation de risque naturel à Douala permet ainsi de tirer des enseignements pour optimiser la préparation, la prévention et la gestion de 
crise d'une part ; et d'autre part le renforcement des politiques locales de gestion du risque naturel.

\section{DISCUSSION}

Cette recherche avait pour objectif la compréhension des mécanismes qui entourent l'adoption des comportements en situation de risque naturel dans la ville de Douala, elle allie les méthodes géographiques de collecte des données et les modèles théoriques en sciences sociales. C'est une démarche scientifique peu empruntée dans les évaluations de la vulnérabilité sociale, elle encourage toutefois une approche de recherche pluridisciplinaire. Les modèles théoriques exploités ici contribuent à comprendre les comportements des ménages en situation de risque naturel à travers l'évaluation des déterminants de la vulnérabilité et de la résilience des sociétés.

Les résultats de cette étude relèvent une connaissance des risques naturels encourus par les ménages, mais à des degrés relatifs d'appréhension. Les ménages sous estiment le risque et tendent à se familiariser avec les évènements naturels. Plusieurs études sur les comportements des populations exposées aux risques naturels sont réalisées dans le monde, peu d'articles scientifiques sont recensés en Afrique. Ces études relèvent que les ménages situés dans les zones à risque tendent à sous évaluer la menace naturelle, mais relativisent leur crainte des phénomènes naturels en fonction des antécédents catastrophiques (Chouari, 2019 ; Tchékémian, 2020). De fortes disparités sont enregistrées dans la perception des risques selon les caractéristiques propres aux ménages (genre, durée de résidence, revenu, éducation). Selon plusieurs études, les femmes auraient une perception des risques différente de celle des hommes (Verlynde, 2018); il existerait une relation significative entre le revenu et la faible perception des risques (Maichanou, 2014 ; Nathan, 2012 ; Lindell et Hwang, 2008) ; une longue durée de résidence dans la zone exposée accroit la perception du risque (Chouari, 2019) ; le degré de perception du risque décroit au fur et à mesure qu'on s'éloigne de l'aléa (Tchékémian, 2020 ; Nathan, 2012).

Rares sont les études qui font appel aux modèles théoriques pour expliquer l'adoption des comportements préventifs en situation de risque. Grothmann et Reusswig (2006) analysent à partir du modèle de la motivation à la protection, les facteurs qui favorisent la mise en place de mesures préventives par les ménages en situation d'inondation à Cologne (Allemagne), ils estiment que le modèle de la motivation à la protection est utile pour expliquer la préparation aux inondations ; Zaalberg et al., (2009) montrent à partir d'une étude réalisée aux Pays Bas, que l'expérience des inondations motive la mise en place de comportements préventifs ; Kouabenan (2007) relève que la motivation des individus à se protéger se fonde sur deux processus cognitifs : l'évaluation de la menace (ou la perception du risque) et l'évaluation de la capacité d'action. Bercera et al., (2013) montrent que les caractéristiques de l'aléa dans les vallées étudiées influencent les représentations du risque et par conséquent les motivations à se protéger ; face au risque majeur, la vulnérabilité sociale se trouve ainsi augmentée.

Les résultats développés dans cet article montrent que l'adoption des comportements préventifs en situation de risque naturel est liée à la perception et aux représentations sociales. Les ménages qui jugent le risque naturel moins sévère, auront tendance à adopter des comportements non préventifs, tandis qu'un risque jugé sévère favorise l'adoption des mesures protectrices. Au-delà de ces considérations, des ménages ayant de grandes motivations à se protéger se retrouvent souvent impuissants en situation de risque naturel à cause de leur faible capacité d'action. En effet, plusieurs recherches relèvent le lien qui existe entre l'adoption des mesures sécuritaires ou préventives et les variables socio-économiques des ménages à partir du modèle des capabilités de Sen, les ménages vulnérables ont une faible capacité d'action et restent impuissants face aux évènements naturels dommageables (Delcroix et Rousseau, 2004 ; Rousseau, 2007 ; Lallau, 2008 ; Bidou et Droy, 2013 ; Benitez et Reghezza, 2018). La priorité des ménages pauvres est l'amélioration de leurs conditions de vie, ils sont les plus vulnérables d'autant plus que les catastrophes naturelles contribuent à l'aggravation de ces vulnérabilités à travers la réduction de leur capacité d'action (Gerrardin et Poirot, 2014). Les ménages pauvres ne peuvent faire signe de résilience que s'ils bénéficient d'un soutien quelconque (Gérardin et Poirot 2017 ; Rousseau, 2013), comme l'amélioration des politiques publiques de gestion des risques naturels ou le renforcement de la cohésion sociale des ménages. 
dans les pays en développement. L'exemple de Douala (Cameroun)

Deux stratégies territoriales sont observées lors des crises environnementales : les mesures de gestion favorisant la protection et des mesures non protectrices. L'adoption des mesures de protection lors des catastrophes naturelles est liée à l'acceptabilité, les paramètres qui concourent au renforcement de ces mesures sont basés sur les degrés d'aléas [Moyen à Fort], et sur une vulnérabilité [Élevée à Très élevée]. Le caractère peu sévère de la catastrophe (pas de pertes en vies humaines) contribue au déni et encourage les ménages exposés à ne pas adopter des comportements préventifs, malgré les pertes matérielles enregistrées. Les ménages qui font le choix d'adopter des mesures non préventives sont motivés par le caractère non sévère des catastrophes naturelles et leur capacité à y faire face. Le refus des risques naturels conduit à adopter des comportements peu protecteurs, le déni n'encourage pas les actions protectrices.

Un autre facteur de la motivation à l'action/inaction trouve son sens dans la nature du danger, les risques naturels inhérents au territoire conduisent les ménages à adopter des comportements préventifs. Par contre, le risque lié à un dysfonctionnement des ouvrages est endossé sur les autorités responsables de l'aménagement urbain. Les ménages dans cette situation tardent à réagir ou attendent la réaction des pouvoirs publics ; ils agissent lorsque leurs biens ou vies sont menacés par l'aléa naturel (cas des ravins liés au dysfonctionnement des buses). Certains ménages se retrouvent à ne rien faire face aux risques naturels, ils sont impuissants pour les uns et pour les autres, le mécanisme perceptif n'aboutit pas à déclencher un comportement préventif.

Au-delà de l'impact peu traumatique des catastrophes naturelles, la passivité de certains ménages s'explique par leur incapacité à faire face aux évènements naturels, une impuissance due à leur situation socio-économique et qui conditionne l'adoption des bonnes pratiques. Les catastrophes naturelles réduisent durablement les capitaux des ménages les plus vulnérables d'où leur difficulté à se reconstituer lors de la phase post-catastrophe. Cette faible capacité d'action des ménages pauvres limite la mise en œuvre des comportements préventifs en situation de risque naturel; au-delà des bénéfices issus de l'adoption des comportements pouvant limiter les chocs négatifs, les ménages pauvres restent passifs lors des catastrophes naturelles et luttent exclusivement pour leur survie car ne possédant pas les capitaux nécessaires pour réagir efficacement.

\section{CONCLUSION}

Lors des situations de catastrophe naturelle, les populations exposées adoptent des comportements préventifs ou non préventifs, ces pratiques sociales sont commandées par un faisceau de facteurs inhérents à l'individu ou au groupe social. À partir d'une étude de cas dans la ville de Douala, cet article a soulevé les motivations à la protection en situation de risque naturel. D'une part, des mesures protectrices sont adoptées lorsque l'individu juge le danger nuisible pour son entourage, cela conduit à une acceptation de la situation catastrophique. D'autre part, le déni de la réalité et la résignation poussent à adopter des comportements non préventifs. Au départ du processus, intervient une information qui est différemment perçue et interprétée par les individus d'où la pluralité des représentations sociales et des comportements qui en découlent.

À ces facteurs, il est indispensable de faire intervenir les variables socio-économiques dans la compréhension des comportements individuels ou collectifs. Les populations installées dans les zones à risque sont majoritairement pauvres et sont plus préoccupées par l'amélioration de leurs conditions de vie. Dans ce cas, la théorie des capabilités (Sen, 1993 ) renseigne que ces individus sont moins dotés en capital et sont plus vulnérables dans la société, d'où la simplification des dangers naturels qui les affectent au quotidien car ils sont bien en deçà de leurs préoccupations économiques. Dans ces conditions de vie, il est difficile pour un ménage d'abandonner son emplacement actuel pour un lieu incertain. Malgré le degré de dangerosité, ce site constitue la principale richesse des ménages pauvres. Au-delà de la simple analyse du processus perception/ comportement en situation de catastrophe, cette étude fournit des informations contextualisées qui peuvent orienter une gestion intégrée des risques naturels. Il ne s'agit aucunement d'une prévision des comportements humains lors des crises futures, loin de là car l'individu réagit en fonction des faits réels ou abstraits dont l'interprétation reste influencée par des facteurs socio-économiques.

Au demeurant, cette étude soulève une question moins actualisée dans les pays en développement, celle du rapport des populations au risque et de la 
communication sur les risques. C'est une dimension essentielle dans toute politique de gestion des risques naturels, elle reste l'élément déclencheur des perceptions et représentations sociales. Une bonne éducation des populations sur les risques naturels qui les menacent est une avancée dans le renforcement de la culture du risque, préalable indispensable à une gestion durable du territoire. Toutes ces initiatives permettront le développement d'une représentation du risque pouvant aboutir au déclenchement des comportements préventifs.

\section{REMERCIEMENTS}

Cet article a été rédigé dans le cadre d'une recherche doctorale $(2016$ - 2019) non soutenue à ce jour. Merci à Olivier ESSOUMAN et Stanislas BETTO, doctorants à l'Université de Douala pour la collecte des données d'enquête. L'auteur tient à remercier Jean Louis YENGUÉ, Professeur des Universités en Géographie (Poitiers, France) et Laurent BRUCKMANN, Assistant à l'Université de Liège (Belgique), pour l'évaluation et les réorientations nécessaires à l'amélioration de cet article.

\section{NOTES}

${ }^{1}$ Ménages dont le niveau de vie est inférieur au seuil de pauvreté. Le seuil de pauvreté est défini à partir du niveau de vie médian (moins de 1000 FCFA/jour, équivalent à 1,53 euros). Cette évaluation est inspirée des travaux de l'institut national de la statistique (ECAM 4 - Enquête camerounaise auprès des ménages).

\section{BIBLIOGRAPHIE}

Abric, J.C. (1994). Pratiques sociales et représentations, Paris : Presses Universitaires de France.

Amanejieu, A. (2018). Analyse temporelle de la représentation du risque d'inondation de 1980 à 2018 à Douala-Cameroun. Mémoire de Master, Université de Liège, $69 \mathrm{p}$.

Armas, I., Ionescu, R., \& Posner, C. N. (2015). Flood risk perception along the Lower Danube river, Romania. Natural Hazards, 79(3), 1913-1931. https:// doi.org/10.1007/s11069-015-1939-8

Barroca, B., Dinardo, M. \& Mboumoua, I. (2013). De la vulnérabilité à la résilience : mutation ou bouleversement ? EchoGéo [En ligne], 24, mis en ligne le 10 juillet 2013, consulté le 07 septembre 2020. URL: http://journals.openedition.org/echogeo/13439

Becerra, S., Peltier, A., Antoine, J.M., Labat, D., Chorda, J., Ribolzi, O., Daupras, F. \& et Dartu,s D. (2013). Comprendre les comportements face à un risque modéré d'inondation. Étude de cas dans le périurbain toulousain (Sud-Ouest de la France). Hydrological Sciences Journal, 58(5), 945-965.

Benitez, F. \& Reghezza, M. (2018). Les capabilités à faire face ou comment repenser la résilience des individus. VertigO - la revue électronique en sciences de l'environnement [En ligne], Hors-série 30 | mai 2018, mis en ligne le 15 mai 2018, consulté le 07 septembre 2020. URL : http://journals.openedition. org/vertigo/19116

Bétard, F. \& Fort, M. (2014). Les risques liés à la nature et leur gestion dans les Suds. Bulletin de l'Association de Géographes Français [En ligne], 91-3 |, mis en ligne le 22 janvier 2018, consulté le 19 avril 2019. URL : http://journals.openedition. org/bagf/1571

Betto, S. (2018). Représentation du risque d'inondation dans le sous bassin versant de Bobongo dans l'arrondissement de Douala $3^{\text {ème }}$. Mémoire de Master de Géographie, Université de Douala, 115p.

Bidou, J.-E. \& Droy, I. (2013). De la vulnérabilité individuelle aux syndromes de vulnérabilité : quelles mesures ? Revue Tiers Monde, 2013/1, 213, 123-142.

Bonnet, C. (2014). Les trois étapes de la perception. Le cerveau de la pensée, Ed. Sciences Humaines, Coll. Synthèse, 213-221.

Boudières, V. (2007). De la gestion des risques, aux risques de la gestion. Géocarrefour [En ligne], 82/12 , mis en ligne le 1 juin 2010, consulté le 16 juillet 2020. URL : http://geocarrefour.revues.org/1448

Bruckmann, L., Amanejieu, A., Zogning, M. \& Ozer, P. (2019). Analyse géohistorique de l'évolution spatio-temporelle du risque d'inondation et de sa gestion dans la zone urbaine de Douala (Cameroun). Physio-Géo [En ligne], 13.

Burningham, K., Fielding, J., \& Thrush, D. (2008). "It'll never happen to me": Understanding public awareness of local flood risk. Disasters, 32(2), 216-238.

Cantin, R. \& Guézo, B. (2020). La résilience des territoires : propositions d'un cadre d'étude systémique. Acta Europeana Systematica, 9(1), 19-34.

Chouari, W. (2019). La perception du risque d'inondations dans les zones inondables du bassin versant de Manouba-Essijoumi (Tunisie nord-orientale) : sensibilité au risque et aux actions de prévention, BSGLg [En ligne], 73 (2019/2). Varia, 117-129 URL: https://popups.uliege.be:443/0770-7576/ index.php?id=5962

Crocq, L. (1994). La psychologie des catastrophes et des blessés psychiques. In Noto R., Huguenard P. et Larcan A. (éds.), Médecine de catastrophe, Paris : Masson, 580p.

D’Ercole, R. (1996). Représentations cartographiques des facteurs de vulnérabilité des populations exposées à une menace volcanique. Application à la région du volcan Cotopaxi (Équateur). Bulletin de l'Institut Français d'Études Andines, 25(3), 479-507. 
dans les pays en développement. L'exemple de Douala (Cameroun)

Dauphiné, A. \& Provitolo, D. (2007). La résilience : un concept pour la gestion des risques. Annales de Géographie, 654, 115-125.

Fogwe, N.Z. \& Tchotsoua, M. (2007). Évaluation géographique de deux décennies de lutte contre les inondations dans la ville de douala (Cameroun). Actes des JSIRAUF, Hanoi, 6-9 novembre 2007, 6p.

Gérardin, H. \& Poirot, J. (2014). Promotion des capabilités dans le cadre des politiques économiques et sociales publiques, communication aux 5èmes Journées du développement du GREThA-CNRS, Le rôle de l'État dans les économies en développement, Université de Bordeaux, 19 et 20 juin.

Gérardin, H. \& Poirot, J. (2017). Résilience et développement durable dans l'optique des capabilités. Les Cahiers de l'Association Tiers-Monde, 32, 21-28.

Gollier, C. (2005). Les déterminants socio-économiques des comportements face aux risques. Revue Économies, 56, 417-21.

Gondard-Delcroix, C. \& Rousseau, S. (2004). Vulnérabilité et Stratégies durables de gestion des risques : Une étude appliquée aux ménages ruraux de Madagascar, Développement durable et territoires [En ligne], Dossier 3 : Les dimensions humaine et sociale du Développement Durable. URL: http://developpementdurable.revues.org/1143

Grothmann, T. \& Reusswig, F. (2006). People at risk of flooding: Why some residents take precautionary action while others do not. Natural Hazards, 38, 101-120.

Hallegatte, S., Vogt-Schilb, A., Bangalore, M. \& Rosenberg J. (2017). Indestructible - Renforcer la résilience des plus pauvres face aux catastrophes naturelles. Licence : Creative Commons Attribution CC BY 3.0 IGO: Washington, DC : La Banque mondiale. GFDDR.

Kouabenan, D.R. (2007). Des croyances aux comportements de protection (deuxième partie). In D.R. Kouabenan, B. Cadet, D. Hermand, \& M.-T. Muñoz-Sastre (éds.), Psychologie du risque : Identifier, évaluer, prévenir. Éditions De Boeck, Collection Ouvertures Psychologiques.

Lallau, B. (2008). Les agriculteurs africains entre vulnérabilité et résilience. Pour une approche par les capabilités de la gestion des risques. Revue Française de Socio-Économie, 2008/1, 177-198.

Lallau, B., Laissus-Benoit, P. \& Mbetid-Bessane, E. (2018). Introduction : la résilience peut-elle passer de la théorie aux pratiques ? Revue internationale des études du développement, 2018/3(235), 9-25.

Lechowska, E. (2018). What determines flood risk perception? A review of factors of flood risk perception and relations between its basic elements. Natural Hazards, 94, 1341-1366.

Leone, F. \& Vinet, F. (dir.) (2006). La vulnérabilité des sociétés et des territoires face aux menaces naturelles. Analyses géographiques. Coll. Géo- risques, Université Paul-Valéry, Montpellier III, vol.1, 140p.

Lindell, M.K. \& Hwang, S.N. (2008). Households' perceived personal risk and responses in a multihazard environment. Risk Analysis, 28(2), 539-556.

Maichanou, A. (2014). Comportement face au risque et risque de comportement : analyse et implications au Niger, Thèse de doctorat, Université de Bordeaux.

Mainet, G. (2005). Douala, de la métropole à la mégapole, croissance et nouvelles dynamiques à l'œuvre. XIe journées de géographie tropicale : les interactions ruptures, transitions et mutations, 7-10 nov. 2005.

Mbaha, J.P., Olinga, J-M. \& Tchiadeu, G. (2013). Cinquante ans de conquête spatiale à Douala : d'héritage colonial en construction à patrimoine socio-spatial vulnérable aux risques naturels. In Actes du Colloque des cinquantenaires, La réunification du Cameroun : Bilans, défis et perspective, FLSH, Université de Douala.

Meva'a, A., Fouda, M. \& Kamwo, M. (2010). Analyse spatiale du risque d'inondation dans le bassin versant du Mbanya à Douala, capitale économique du Cameroun, Novatech, 10p.

Meva'a, A. \& Fouda, M. (2018). Répercussions environnementales du ravinement à Sodikombo (Douala) -Cameroun liées à l'émergence urbaine. In Méva'a A. \& Fogwe Z. (eds), DOUALA - FUTURIS/ Tome 2 : Défis et enjeux de l'émergence urbaine entre Environnement et Développement Durable. Éditions Cheikh Anta Diop, 338-364.

Mounde, A. (2019). Douala : de la construction de la vulnérabilité à la catastrophe programmée. In Catastrophes naturelles : résilience à l'ère des changements climatiques. LIAISON - ENERGIE FRANCOPHONIE, 17-18.

Nathan, F. (2012). Perception du risque, vulnérabilité et résilience des foyers. In La régulation sociale des risques de catastrophe : Ethnographie des quartiers périphériques de La Paz. Genève : Graduate Institute Publication.

Olinga, J.M. (2012). Vulnérabilité des espaces urbains et stratégies locales de développement durable: Étude du cas de la ville de Douala (Cameroun), Mémoire de Master de Géographie, Université de Douala, 145p.

Quenault, B. (2015). La vulnérabilité, un concept central de l'analyse des risques urbains en lien avec le changement climatique. Les Annales de la recherche urbaine, 110, 138-151.

Rogers, R.W. (1983). Cognitive and physiological processes in fear appeals and attitude change: a revised theory of protection motivation. In J. Cacioppo \& R. Petty (Eds.), Social psychophysiology. New York: Guilford Press.

Rousseau, S. (2013). Capabilités, risques et vulnérabilité. In Dubois J.-L., Lachaud J.-P., Montaud J.-M., 
Pouille A. (dir.), Pauvreté et développement socialement durable, Bordeaux : P.U. de Bordeaux, 11-22.

Saha, F., Nkemta, D., Tchnindjang, M., Voundi, E., \& Mbevo, P. (2018). Production des risques dits « naturels » dans les grands centres urbains du Cameroun. Natures Sciences Sociétés, 26(4), 418-433.

Sen, A. (1993). Capability and Well-Being. In M.C Nussbaum and A. Sen (eds), The Quality of Life, Oxford : Clarendon Press, 30-53.

Tadonki, G. (1999). Douala: Les exclus des marécages. Yaoundé : Éditions Mandara, 112p.

Tchékémian, A. (2020). Appréhension de la notion de risques et ses différentes déclinaisons en géographie. Risques urbains, ISTE Ltd.

Tchiadeu, G. \& Olinga, J.M. (2012). La ville de Douala : entre baisse des précipitations et hausse des températures. In : Les climats régionaux : observation et modélisation, 25ème colloque de l'Association Internationale de Climatologie (Grenoble), S. BIGOT et S. ROME édit., Édit. AIC / LTHE, 727-732

Tchounga, G. (2020). Gouvernance territoriale des risques naturels : Une approche de recherche multirisque sur le littoral camerounais (Cas du département du Wouri). Thèse de Doctorat non soutenue. Département de géographie, Université de Douala, 338p.

Thouret, J.C. (2003). Évaluation, prévention et gestion des risques naturels dans les villes d'Amérique latine, in Y. Veyret (dir.). Les risques, Paris : Sedes, Coll. Dossiers des images économiques du Monde, 63-87. Verlynde, N. (2018). De la perception du risque d'inondation aux propositions d'adaptation en territoire de côtes basses densément peuplées : le cas de la communauté urbaine de Dunkerque. Université du Littoral Côte d'Opale.

Zaalberg, R., Midden, C., Meijnders, A. \& McCalley, T. (2009). Prevention, adaptation, and threat denial: Flooding experiences in the Netherlands. Risk Analysis, 29(12), 1759-1778.

Zogning, M., Tonye, E., Ambara, G. \& Tsalefack, M. (2013). Cartography of flood prone areas and assessment of flooding housing in Douala (Cameroon). Actes de la conférence «Disaster risk identification, assessment and monitoring », Éd. Nations Unies, en ligne : https://www.researchgate.net/publica$\underline{\text { tion/273321869 }}$

\section{Coordonnées de l'auteur :}

Gille Baustert TCHOUNGA

Département de Géographie Université de Douala gtchounga@yahoo.fr 